\title{
DOMINATION IN GRAPHS OF MINIMUM DEGREE FOUR
}

\author{
Moo Young Sohn and Yuan Xudong
}

\begin{abstract}
A dominating set for a graph $G$ is a set $D$ of vertices of $G$ such that every vertex of $G$ not in $D$ is adjacent to a vertex of $D$. Reed [11] considered the domination problem for graphs with minimum degree at least three. He showed that any graph $G$ of minimum degree at least three contains a dominating set $D$ of size at most $\frac{3}{8}|V(G)|$ by introducing a covering by vertex disjoint paths. In this paper, by using this technique, we show that every graph on $n$ vertices of minimum degree at least four contains a dominating set $D$ of size at most $\frac{4}{11}|V(G)|$.
\end{abstract}

\section{Introduction}

Throughout this paper, by a graph $G$ we always mean a finite, undirected, and simple graph with vertex set $V(G)$ and edge set $E(G)$. For $x, y \in V(G)$, $x y$ denotes the edge with ends $x$ and $y$. If $x y \in E(G)$, we say that $y$ is a neighbor of $x$ or $y$ is joined to $x$, and denote by $N(x)$ the set of neighbors of $x$. $d(x)=|N(x)|$ is called the degree of $x$. A subgraph $H$ is said to be induced by $U$ if $V(H)=U$ and $x y \in E(H)$ if and only if $x y \in E(G), x, y \in U$. A set $D$ of vertices of a graph $G$ is called a dominating set if every vertex of $V(G)-D$ is adjacent to at least one element of $D$. The domination number of $G$, denoted by $\gamma(G)$, is the minimum cardinality of a dominating set of $G$. It has been proved [5] that the decision problem corresponding to the domination number for arbitrary graphs is $N P$-complete. Thus, the exploration of lower and upper bounds for the domination number as sharp as possible is of great significance. Many results on upper bounds on the domination number in terms of some basic parameters such as the numbers of vertices and edges, the minimum and maximum degree and so on, have been obtained. The terminologies not presented here can be found in [6].

Let $\delta=\delta(G)$ denote the minimum degree of graph $G$. An early result of Ore (see [9]) states that $\gamma(G) \leq \frac{n}{2}$ if $G$ is a graph of order $n$ with the minimum degree at least one. This result was improved to $\gamma(G) \leq \frac{2 n}{5}$ by McCuaig and Shepherd in [8] for the connected graph $G$ which has minimum degree at least

Received September 28, 2007.

2000 Mathematics Subject Classification. 05C50, 05 C69.

Key words and phrases. graphs, domination number.

This research is financially supported by Changwon National University in 2006. 
two and is not one of seven exceptional graphs. Reed in [11] considered the case for the graphs with minimum degree at least three, and obtained that $\gamma(G) \leq \frac{3 n}{8}$. In this direction, an obvious conjecture (see [6]) seems to be that for any graph $G$ with $\delta(G) \geq k, \gamma(G) \leq \frac{k}{3 k-1} n$. However, Caro and Roditty (see [2], [3] and also [1]) proved that for any graph $G$ with minimum degree $\delta, \gamma(G) \leq n\left[1-\delta\left(\frac{1}{\delta+1}\right)^{1+\frac{1}{\delta}}\right]$. For $\delta(G) \geq 7$, it is easy to verify that $n\left[1-\delta\left(\frac{1}{\delta+1}\right)^{1+\frac{1}{\delta}}\right]<\frac{\delta}{3 \delta-1} n$ by using calculus. Thus, the question remains open only for graphs $G$ with $4 \leq \delta(G) \leq 6$. The purpose of this paper is to give a positive answer for the graph $G$ with minimum degree $\delta(G) \geq 4$.

Main Theorem. Let $G$ be a graph of order $n$ with minimum degree at least four. Then

$$
\gamma(G) \leq \frac{4}{11} n
$$

The proof of Main Theorem is completed by choosing a dominating set $D$ of $G$ based on the so-called vertex disjoint paths cover, which was introduced by Reed [11]. By cases analysis, we prove three basic facts, from these, $|D| \leq \frac{4}{11} n$ is obtained. For convenience, we use $|G|$ for the number of vertices of the graph $G$.

A cover of vertex disjoint paths of $G$, or simply a $v d p$-cover, is a set of vertex disjoint paths $P_{1}, \ldots, P_{k}$ such that $V(G)=V\left(P_{1}\right) \cup \cdots \cup V\left(P_{k}\right)$. A path $P$ is called a 0 -, 1- or 2-path if $|P|$ is congruent to 0,1 or $2 \bmod 3$, respectively. For a $v d p$-cover $S$ of $G$, let $S_{i}(i=0,1,2)$ be the set of $i$-paths in $S$. If $P=P^{\prime} x P^{\prime \prime}$, where $P^{\prime}$ is an $i$-path and $P^{\prime \prime}$ is a $j$-path (and $x$ is on neither of those paths), then we say $x$ is an $(i, j)$-vertex of $P$. Let $P \in S$ and $x$ be an endvertex of $P$. We say that $x$ is an out-endvertex if it has a neighbor which is not on $P$. If $P$ is a 2 -path, we say that $x$ is a $(2,2)$-endvertex if it is not an out-endvertex and is adjacent to some $(2,2)$-vertex of $P$.

\section{Choose a dominating set}

In the below, we always assume that $G$ is a graph of order $n$ with $\delta(G) \geq 4$. For convenience, we assume that $G$ is connected. We first choose a $v d p$-cover $S$ of $G$ such that

(1) $2\left|S_{1}\right|+\left|S_{2}\right|$ is minimized,

(2) Subject to (1), $\left|S_{2}\right|$ is minimized,

(3) Subject to $(2), \sum_{P_{i} \in S_{0}}\left|P_{i}\right|$ is minimized,

(4) Subject to (3), $\sum_{P_{i} \in S_{1}}\left|P_{i}\right|$ is minimized.

Let $x$ be an out-endvertex of $P_{i} \in S_{1} \cup S_{2}, y$ a neighbor of $x$ on some path $P_{j}$ distinct from $P_{i}$. Let $P_{j}=P_{j}^{\prime} y P_{j}^{\prime \prime}$. Then, we have the following assertion (for the proof, see [11], Observation 1-3).

Assertion 1. $P_{j}$ is not a 1-path. If $P_{j}$ is a 0-path, then both $P_{j}^{\prime}$ and $P_{j}^{\prime \prime}$ are 1-paths; if $P_{j}$ is a 2-path, then both $P_{j}^{\prime}$ and $P_{j}^{\prime \prime}$ are 2-paths. 
Having chosen the minimal $v d p$-cover $S=\left\{P_{1}, \ldots, P_{k}\right\}$, we rearrange the paths of $S$ to obtain a new $v d p$-cover $S^{\prime}=\left\{P_{1}^{\prime}, \ldots, P_{k}^{\prime}\right\}$ such that $P_{i}^{\prime}$ is a Hamilton path on $V\left(P_{i}\right)$, and so that the number of out-endvertices is maximized, and subject to this, the number of $(2,2)$-endvertices is maximized. Clearly, $S^{\prime}$ is still minimal with respect to the above four conditions. For convenience, we still denote the new $v d p$-cover of $G$ by $S$.

Now, for each 1-path $P$ in $S$ which has an out-endvertex we choose some vertex $y \notin P$ which is adjacent to the endvertex of $P$. We say that $y$ is the acceptor for $P$. For each 2-path $P$ in $S$ which has two out-endvertices, for each of these endvertices we choose a vertex of $G-P$ which is adjacent to it and designate it as the acceptor corresponding to that endvertex. For each 2-path $P$ in $S$ which has precisely one out-endvertex $x$ and $|P| \leq 8$, we choose some vertex $y \notin P$ which is adjacent to it and designate it as the acceptor for $P$. We call a path in $S$ accepting if it contains an acceptor. We next specify a set $A \subseteq S$ of 2-paths. Initially, let $A$ be the set of accepting 2-paths. While there is any out-endvertex $x$ of a path in $A$ for which we have not chosen an acceptor, we choose a neighbor of this endvertex in $G-P$ and designate it as an acceptor for $x$. If this new acceptor is on a previously non-accepting 2-path $P^{\prime}$, then we add $P^{\prime}$ to $A$. We continue this process until there is an acceptor for every out-endvertex of the paths in $A$. In addition, for any $(2,2)$-endvertex $x$ of any path $P$ in $A$, we choose a $(2,2)$-vertex $y$ of $P$ which is adjacent to $x$ and designate it as an inacceptor for $x$.

For any accepting 2-path $P$, we partition $P=P_{1} P_{2} P_{3}$ such that $P_{1}$ and $P_{3}$ are both 1-paths which contain neither acceptors nor inacceptors, and maximal with this property. We say that $P_{1}$ and $P_{3}$ are tips of $P$ and $P_{2}$ is its central path. By the maximality of $P_{1}, P_{3}$ and Assertion 1 , if $x \in P_{2}$ is adjacent in $P_{2}$ to an endvertex of $P_{2}$, then it is an acceptor or inacceptor.

Before the description of choosing the dominating set, we present the following fact.

Assertion 2. Let $P \in S$ be a 2-path. If $P$ has precisely one out-endvertex $x$ and $|P| \leq 8$, then $V(P)$ has a subset of $\left\lfloor\frac{|P|}{3}\right\rfloor$ vertices which dominate $V(P)-x$.

We will prove Assertion 2 in next section. Now, we choose a dominating set $D$ of $G$ in the following manner:

Step 1: For each 0 -path $P$, we put every $(1,1)$-vertex of $P$ in $D$.

Step 2: For each accepting 2-path $P$, we put into $D$ every $(2,2)$-vertex of $P$ which is in the central path of $P$.

Step 3: For each 1-path $P$ with at least one out-endvertex, we choose $\left\lfloor\frac{|P|}{3}\right\rfloor$ vertices of $P$ which dominate all of the vertices of $P$ except for the endvertex of $P$ which is adjacent to the acceptor of $P$. We put these vertices in $D$. For each non-accepting 2-path $P$ with two out-endvertices we choose $\left\lfloor\frac{|P|}{3}\right\rfloor$ vertices of $P$ which dominate its interior vertices. We put these vertices in $D$. For each 
non-accepting 2-path $P$ which has precisely one out-endvertex $x$ and $|P| \leq 8$, we choose $\left\lfloor\frac{|P|}{3}\right\rfloor$ vertices of $P$ which dominate all of the vertices of $P$ except for the endvertex $x$ (By Assertion 2, we can do that). We put these vertices in $D$.

Step 4: For each 1-path $P$ with no out-endvertex, we choose a subset of $V(P)$ which dominate $V(P)$ to put in $D$. If possible, we choose a set of $\left\lfloor\frac{|P|}{3}\right\rfloor$ vertices; otherwise we choose a set of $\left\lceil\frac{|P|}{3}\right\rceil$ vertices. For each non-accepting 2-path $P$ which has no out-endvertex, or has precisely one out-endvertex and $|P| \geq 11$, we choose a subset of $V(P)$ which dominate $V(P)$ to put in $D$. If possible, we choose a set of $\left\lfloor\frac{|P|}{3}\right\rfloor$ vertices, otherwise we choose a set of $\left\lceil\frac{|P|}{3}\right\rceil$ vertices.

Step 5: For each tip $P_{1}$ of an accepting 2-path $P$, if the common endvertex $x$ of $P_{1}$ and $P$ is adjacent to a vertex chosen in Step 1 or 2 , we choose $\left\lfloor\frac{\left|P_{1}\right|}{3}\right\rfloor$ of vertices of $P_{1}$ which dominate the remaining vertices of $P_{1}$ and put them in $D$. If $x$ is not adjacent to a vertex chosen in Step 1 or 2 , we choose a set which dominates $P_{1}$ to put in $D$. If possible, we choose $\left\lfloor\frac{\left|P_{1}\right|}{3}\right\rfloor$ vertices, otherwise we choose $\left\lceil\frac{\left|P_{1}\right|}{3}\right\rceil$ vertices.

It is easy to see that $D$ is a dominating set of $G$ (or see [11], Observation 5-8). To calculate the size of $D$, we define the following sets.

(i) $O_{1}$ : the set of 1-paths $P$ which either have an out-endvertex or contain a dominating set of size $\left\lfloor\frac{|P|}{3}\right\rfloor$.

(ii) $\mathrm{O}_{2}$ : the set of non-accepting 2-paths $P$ which have two out-endvertices or contain a set of size $\left\lfloor\frac{|P|}{3}\right\rfloor$ that dominates all of the vertices of $P$, and all non-accetping 2-paths which have precisely one out-endvertex and $|P| \leq 8$.

(iii) $I_{1}$ : the set of 1 -paths not in $O_{1}$.

(iv) $\mathrm{I}_{2}$ : the set of non-accepting 2-paths not in $\mathrm{O}_{2}$.

(v) $E$ : the set of such tips $P_{1}$ of an accepting 2-path $P$, which is in $E$ if and only if the corresponding endvertex of $P$ is neither an out-endvertex nor a $(2,2)$-endvertex and we cannot dominate $P_{1}$ using $\left\lfloor\frac{\left|P_{1}\right|}{3}\right\rfloor$ vertices.

(vi) $W$ : the set of $(2,2)$-endvertices of accepting 2-paths for which we have chosen an inacceptor.

Then

$$
\begin{aligned}
|D|= & \sum_{P \in O_{1}} \frac{|P|-1}{3}+\sum_{P \in O_{2}} \frac{|P|-2}{3}+\sum_{P \in I_{1}} \frac{|P|+2}{3}+\sum_{P \in I_{2}} \frac{|P|+1}{3} \\
& +\sum_{P \in S_{0}} \frac{|P|}{3}+\sum_{P \in A} \frac{|P|-2}{3}+|E| .
\end{aligned}
$$

Equivalently,

$$
|D|=\frac{n}{3}-\frac{1}{3}\left|O_{1}\right|-\frac{2}{3}\left|O_{2}\right|+\frac{2}{3}\left|I_{1}\right|+\frac{1}{3}\left|I_{2}\right|-\frac{2}{3}|A|+|E| .
$$

Note that each accepting 2-path corresponds to an endvertex of some path in $O_{1} \cup O_{2}$ or to an endvertex of an accepting 2-path of $A$ which is not in 
$E \cup W$. Thus, we have $|A| \leq\left|O_{1}\right|+2\left|O_{2}\right|+2|A|-|E|-|W|$, and so $|E| \leq$ $\left|O_{1}\right|+2\left|O_{2}\right|+|A|-|W|$. Also, $|E| \leq 2|A|-|W|$. Thus,

$$
|D| \leq \frac{n}{3}+\frac{2}{3}\left|I_{1}\right|+\frac{1}{3}\left|I_{2}\right|+\frac{|E|}{2}-\frac{|W|}{2} .
$$

For each element $T$ of $E$, there is an accepting 2-path $P_{T}$ such that $T$ is a tip of $P_{T}$. Now we define $E^{\prime} \subseteq E$ by saying that each $T \in E$ is in $E^{\prime}$ if the endpoint of $P_{T}$ not in $T$ is not an element of $W$.

Clearly, $\left|E^{\prime}\right| \geq|E|-|W|$, and so

$$
|D| \leq \frac{n}{3}+\frac{2}{3}\left|I_{1}\right|+\frac{1}{3}\left|I_{2}\right|+\frac{1}{2}\left|E^{\prime}\right|
$$

In next section, we will prove some facts (Lemma 3, Lemma 4, Lemma 5), with $(*)$ that imply $|D| \leq \frac{4}{11} n$.

\section{1-paths with short length}

Essentially, in this section we will prove that every 1-path $P$ of short length with some additional conditions can be dominated by $\left\lfloor\frac{|P|}{3}\right\rfloor$ vertices. We will assume the same conditions and use the notations as in last section. We first state three simple observations.

$\left(Q_{1}\right)$ Let $P=x_{1} x_{2} \cdots x_{3 k+1}(k \geq 1)$ be a 1 -path. If $x_{1}$ is adjacent to a vertex $x_{3 i}$ for some $1 \leq i \leq k$, then $P$ can be dominated by $k$ vertices.

$\left(Q_{2}\right)$ Let $C$ be a cycle of $3 k+1(k \geq 1)$ vertices, $B=b_{1} b_{2} b_{3}$ be a path such that $V(C) \cap V(B)=\emptyset$. If $b_{2}$ has a neighbor in $C$, then $V(C) \cup V(B)$ can be dominated by $k+1$ vertices.

$\left(Q_{3}\right)$ Let $P=x_{1} x_{2} \cdots x_{3 k-1}(k \geq 1)$ be a path, and $x \notin P$. If $x$ is adjacent to some vertex of $\cup_{i=1}^{k}\left\{x_{3 i-2}, x_{3 i-1}\right\}$, then $V(P) \cup\{x\}$ can be dominated by $k$ vertices.

Next we show two technical results.

Lemma 1. Let $C=x_{1} x_{2} \cdots x_{3 k+1} x_{1}(1 \leq k \leq 4)$ be a cycle of $G$, $H$ the subgraph induced by $V(C)$. If $N\left(x_{i}\right) \subseteq V(C)$ for any $x_{i} \in V(C)$ such that there is a Hamilton path from $x_{i}$ to $x_{3 k+1}$ in $H$, then $H$ has a dominating set of $k$ vertices.

Proof. Assume to the contrary that $H$ has no any dominating set of $k$ vertices. We only prove for $k=4$. For $k \leq 3$ we can deduce a contradiction by the same reasoning.

Let $k=4$ and $C=x_{1} x_{2} \cdots x_{13} x_{1}$. Then, both $x_{1}, x_{12}$ are the endvertices of some Hamilton paths to $x_{13}$ in $H$, thus $N\left(x_{1}\right) \subseteq V(C)$ and $N\left(x_{12}\right) \subseteq V(C)$. First we check the possible neighbors of $x_{1}$. By $\left(Q_{1}\right), x_{1}$ is not adjacent to any of $x_{3}, x_{6}, x_{9}, x_{12}$. If $x_{1}$ is adjacent to $x_{10}$, as $x_{12}$ has a neighbor in the cycle $C^{\prime}=x_{1} x_{2} \cdots x_{10} x_{1}$, by $\left(Q_{2}\right)$, then $H$ has a dominating set of four vertices, a contradiction. On the other hand, if $x_{12}$ is adjacent to both $x_{8}$ and $x_{9}$, then $x_{10}$ is an endvertex of a Hamilton path of $H$ to $x_{13}$, by applying $\left(Q_{2}\right)$ 
to $x_{9} x_{10} x_{11}$ and the cycle $x_{1} x_{2} \cdots x_{8} x_{12} x_{13} x_{1}$, then $H$ has a dominating set of four vertices, a contradiction. Thus, if $x_{1}$ is adjacent to $x_{7}$, applying $\left(Q_{2}\right)$ to $x_{11} x_{12} x_{13}$ and the cycle $x_{1} x_{2} \cdots x_{7} x_{1}, x_{12}$ is not adjacent to any vertex of the cycle $x_{1} x_{2} \ldots x_{7} x_{1}$. So we can deduce that $x_{12}$ is adjacent to both $x_{8}$ and $x_{9}$, a contradiction.

Hence, $x_{1}$ has extra neighbors only in $\left\{x_{4}, x_{5}, x_{8}, x_{11}\right\}$. Symmetrically, $x_{12}$ has extra neighbors only in $\left\{x_{9}, x_{8}, x_{5}, x_{2}\right\}$. Now, if $x_{1}$ is adjacent to $x_{4}$, by $\left(Q_{2}\right)$, then $x_{12}$ is not adjacent to $x_{2}$. As $x_{12}$ is not adjacent to both $x_{8}$ and $x_{9}$, $x_{12}$ is adjacent to $x_{5}$. Note that, $x_{3}$ is an endvertex of a Hamilton path of $H$ to $x_{13}$. As $x_{1}$ dominates $x_{2}, x_{4}, x_{13}, H$ has a dominating set of four vertices for any choice of the neighbor of $x_{3}$ in the 8-cycle $C^{\prime \prime}=x_{5} x_{6} \cdots x_{12} x_{5}$, a contradiction. So, $x_{1}$ is not adjacent to $x_{4}$, and symmetrically, $x_{12}$ is not adjacent to $x_{9}$. If $x_{1}$ is adjacent to $x_{11}$, then $x_{10}$ is an endvertex of a Hamilton path of $H$ to $x_{13}$. Thus, $x_{12}$ is still not adjacent to $x_{8}$, for otherwise, we apply $\left(Q_{2}\right)$ to $x_{9} x_{10} x_{11}$ and the cycle $x_{1} x_{2} \cdots x_{8} x_{12} x_{13} x_{1}$ to obtain a contradiction. So, $x_{12}$ is adjacent to both $x_{2}, x_{5}$. Then, $x_{3}$ is an endvertex of a Hamilton path of $H$ to $x_{13}$. By applying $\left(Q_{2}\right)$ to $x_{2} x_{3} x_{4}$ and the cycle $x_{1} x_{11} x_{10} \cdots x_{5} x_{12} x_{13} x_{1}$, also a contradiction. Hence, $x_{1}$ is adjacent only to both $x_{5}$ and $x_{8}$, and symmetrically, $x_{12}$ is also adjacent to only both $x_{8}, x_{5}$. Then, $x_{7}$ is an endvertex of a Hamilton path of $H$ to $x_{13}$. By $\left(Q_{1}\right)$ and $\left(Q_{2}\right), x_{7}$ is adjacent to neither $x_{9}$ nor any vertex of the cycle $x_{1} \cdots x_{5} x_{12} x_{13} x_{1}$. Thus, $x_{7}$ is adjacent to $x_{10}$. Then, $\left\{x_{2}, x_{5}, x_{10}, x_{12}\right\}$ dominates $H$, a contradiction. This proves Lemma 1.

Lemma 2. Let $C=x_{1} x_{2} \cdots x_{3 k+2} x_{1}(1 \leq k \leq 4)$ be a cycle of $G$, $H$ the subgraph induced by $V(C)$. If $N\left(x_{i}\right) \subseteq V(C)$ for any $x_{i} \in V(C)$ such that there is a Hamilton path from $x_{i}$ to $x_{3 k+2}$ in $H$, then $V(C)-\left\{x_{3 k+2}\right\}$ can be dominated by $k$ vertices.

Proof. Assume to the contrary that $V(C)-\left\{x_{3 k+2}\right\}$ can not be dominated by $k$ vertices. We still prove only for $k=4$ and omit for $k \leq 3$.

Let $k=4$ and $C=x_{1} x_{2} \cdots x_{14} x_{1}$. Then, both $x_{1}, x_{13}$ are the endvertices of some Hamilton paths to $x_{14}$ in $H$, thus $N\left(x_{1}\right) \subseteq V(C)$ and $N\left(x_{13}\right) \subseteq$ $V(C)$. Note that, $x_{1}$ and $x_{13}$ are symmetrical. By $\left(Q_{1}\right), x_{1}$ is not adjacent to any of $x_{3}, x_{6}, x_{9}, x_{12}$. If $x_{1}$ is adjacent to both $x_{4}, x_{5}$, then both $x_{2}, x_{3}$ are the endvertices of some hamiltonian paths of $H$ to $x_{14}$. As $x_{1}$ dominates $x_{2}, x_{4}, x_{5}$, by $\left(Q_{3}\right), x_{3}$ has extra neighbors only in $\left\{x_{5}, x_{8}, x_{11}, x_{14}\right\}$. On the other hand, as $x_{4}$ dominates $x_{1}, x_{3}, x_{5}$, by $\left(Q_{3}\right), x_{2}$ has at least one neighbor in $\left\{x_{5}, x_{8}, x_{11}, x_{14}\right\}$. Hence, $\left\{x_{5}, x_{8}, x_{11}, x_{14}\right\}$ dominates $V(C)-\left\{x_{3 k+2}\right\}$, a contradiction. So, $x_{1}$ is not adjacent to both $x_{4}, x_{5}$, and symmetrically, $x_{13}$ is not adjacent to both $x_{9}, x_{10}$. In the sequel, we distinguish five cases according to the possible neighbors of $x_{1}$.

1. $x_{1}$ is adjacent to $x_{13}$. Then, both $x_{2}$ and $x_{12}$ are the endvertices of Hamilton paths to $x_{14}$ in $H$. As $x_{1}$ has one more neighbor in $\left\{x_{4}, x_{5}, x_{7}, x_{8}, x_{10}, x_{11}\right\}$, we consider the following subcases. 
(1.1) $x_{1}$ is adjacent to $x_{4}$. Then, $x_{3}$ is an endvertex of Hamilton path to $x_{14}$ in $H$. As $x_{1}$ dominates $x_{2}, x_{4}$ and $x_{13}$, by $\left(Q_{3}\right), x_{3}$ has extra neighbors only in $\left\{x_{7}, x_{10}, x_{13}, x_{14}\right\}$. If $x_{3}$ is adjacent to $x_{10}$, as $N\left(x_{12}\right) \subseteq V(C)$, we apply $\left(Q_{2}\right)$ to $x_{11} x_{12} x_{13}$ and the cycle $x_{1} x_{4} x_{5} \cdots x_{10} x_{3} x_{2} x_{1}$, then there is a dominating set of four vertices of $V(C)-\left\{x_{14}\right\}$, a contradiction. If $x_{3}$ is adjacent to $x_{13}$, then $x_{13}$ dominates $x_{1}, x_{3}$ and $x_{12}$. As $N\left(x_{2}\right) \subseteq V(C)$, still by $\left(Q_{3}\right)$, then $x_{2}$ has extra neighbors only in $\left\{x_{6}, x_{9}, x_{12}, x_{14}\right\}$, and thus $x_{2}$ is adjacent to at least one vertex of $\left\{x_{6}, x_{9}, x_{12}\right\}$. Then, $\left\{x_{4}, x_{6}, x_{9}, x_{12}\right\}$ dominates $V(C)-\left\{x_{14}\right\}$, a contradiction. Hence, $x_{3}$ is adjacent to $x_{14}$. On the other hand, as $x_{4}$ dominates $x_{1}, x_{3}$ and $x_{5}$, by $\left(Q_{3}\right), x_{2}$ has extra neighbors only in $D^{\prime}:=\left\{x_{5}, x_{8}, x_{11}, x_{14}\right\}$. Then, $D^{\prime}$ dominates $V(C)-\left\{x_{14}\right\}$, a contradiction.

(1.2) $x_{1}$ is adjacent to $x_{10}$. As $N\left(x_{12}\right) \subseteq V(C)$, by applying $\left(Q_{2}\right)$ to $x_{11} x_{12} x_{13}$ and the cycle $x_{1} x_{2} \cdots x_{10} x_{1}$, we have that four vertices dominate $V(C)-\left\{x_{14}\right\}$, a contradiction.

(1.3) $x_{1}$ is adjacent to $x_{5}$. By (1.1) and (1.2), assume that both $x_{1}, x_{13}$ are not adjacent to $x_{4}$ and $x_{10}$. Now $x_{4}$ is an endvertex of Hamilton path to $x_{14}$ in $H$, we have $N\left(x_{4}\right) \subseteq V(C)$. By $\left(Q_{1}\right)$ and above result, $x_{4}$ has extra neighbors only in $\left\{x_{7}, x_{8}, x_{10}, x_{11}, x_{14}\right\}$. If $x_{4}$ is adjacent to both $x_{7}$ and $x_{8}$, then $x_{6}$ is an endvertex of a Hamilton path of $H$ to $x_{14}$, and thus, by applying $\left(Q_{2}\right)$ to $x_{5} x_{6} x_{7}$ and the cycle $x_{1} \cdots x_{4} x_{8} \cdots x_{13} x_{1}$, we have a contradiction. If $x_{4}$ is adjacent to $x_{10}$, then we have a 10-cycle without three vertices $\left\{x_{11}, x_{12}, x_{13}\right\}$, thus, by $\left(Q_{2}\right)$, we also have a contradiction. If $x_{4}$ is adjacent to $x_{14}$, then $x_{3} x_{2} x_{1} x_{13} \cdots x_{4} x_{14}$ is a Hamilton path of $H$, and thus $N\left(x_{3}\right) \subseteq V(C)$. By applying $\left(Q_{2}\right)$ to $x_{2} x_{3} x_{4}$ and the cycle $x_{1} x_{5} \cdots x_{13} x_{1}$, we have a contradiction. Summarizing, $x_{4}$ must be adjacent to $x_{11}$. Then, $x_{11}$ dominates $x_{10}, x_{12}, x_{4}$. By applying $\left(Q_{3}\right)$ to $x_{13}$ and the path $x_{3} x_{2} x_{1} x_{5} \cdots x_{9}$, we have that $x_{13}$ must be adjacent to $x_{7}$. As $N\left(x_{2}\right) \subseteq V(C)$, by $\left(Q_{2}\right), x_{2}$ has no neighbor in the cycle $x_{7} x_{8} \cdots x_{13} x_{7}$. Again by $\left(Q_{1}\right), x_{2}$ has two extra neighbors only in $\left\{x_{5}, x_{6}, x_{14}\right\}$. If $x_{2}$ is adjacent to $x_{14}$, then $x_{3}$ is also an endvertex of a Hamilton path of $H$ to $x_{14}$, by using the same reasoning as above, we have a contradiction. Thus, $x_{2}$ is adjacent to both $x_{5}, x_{6}$. By applying $\left(Q_{2}\right)$ to $x_{3} x_{4} x_{5}$ and the cycle $x_{1} x_{2} x_{6} \cdots x_{13} x_{1}$, we have a contradiction.

(1.4) $x_{1}$ is adjacent to $x_{7}$. Then, by $\left(Q_{2}\right)$ and $\left(Q_{1}\right), x_{12}$ has extra neighbors only in $\left\{x_{8}, x_{9}, x_{14}\right\}$. If $x_{12}$ is adjacent to both $x_{8}$ and $x_{9}$, then $x_{10}$ is also an endvertex of a Hamilton path of $H$ to $x_{14}$, and thus $N\left(x_{10}\right) \subseteq V(C)$. By applying $\left(Q_{2}\right)$ to $x_{9} x_{10} x_{11}$ and the cycle $x_{1} \cdots x_{8} x_{12} x_{13} x_{1}$, we have a contradiction. So, $x_{12}$ must be adjacent to $x_{14}$ and one of $x_{8}, x_{9}$. In this case $x_{12} \cdots x_{1} x_{13} x_{14} x_{12}$ is a Hamilton cycle and that $x_{12}, x_{13}$ are adjacent. Note that $x_{12}$ is adjacent to $x_{8}$ or $x_{9}$, this is the same situation as (1.1) or (1.3), a contradiction.

(1.5) $x_{1}$ is adjacent to $x_{8}$ or $x_{11}$. By symmetry and (1.1)-(1.4), $x_{13}$ has one more neighbor only in $\left\{x_{3}, x_{6}\right\}$. Then $\left\{x_{3}, x_{6}, x_{8}, x_{11}\right\}$ dominates $V(C)-\left\{x_{14}\right\}$, a contradiction. 
2. $x_{1}$ is adjacent to $x_{11}$. By $1, x_{1}, x_{13}$ are not adjacent. As $x_{11}$ dominates $\left\{x_{1}, x_{10}, x_{12}\right\}$, by $\left(Q_{3}\right), x_{13}$ has extra neighbors only in $\left\{x_{4}, x_{7}, x_{10}\right\}$.

If $x_{13}$ is adjacent to $x_{10}$, then $x_{12}$ is an endvertex of a Hamilton path of $H$ to $x_{14}$. As $x_{10}$ dominates $\left\{x_{9}, x_{11}, x_{13}\right\}$, still by $\left(Q_{3}\right), x_{12}$ has extra neighbors only in $\left\{x_{3}, x_{6}, x_{9}, x_{14}\right\}$. If $x_{12}$ is adjacent to $x_{14}$, then it is the same situation as 1 , a contradiction. If $x_{12}$ is adjacent to $x_{9}$, by noting that $x_{13}$ has one more neighbor in $\left\{x_{4}, x_{7}\right\}$, then $\left\{x_{1}, x_{4}, x_{7}, x_{9}\right\}$ dominates $V(C)-\left\{x_{14}\right\}$, a contradiction. If $x_{12}$ is adjacent to both $x_{3}, x_{6}$, then there is a 10-cycle $x_{3} \cdots x_{10} x_{13} x_{12} x_{3}$ which excludes $x_{2} x_{1} x_{11}$. As $x_{1}$ has one more neighbor in this cycle, by $\left(Q_{2}\right)$, we also have a contradiction.

Otherwise, $x_{13}$ is adjacent to both $x_{4}$ and $x_{7}$. Then, $x_{5}$ is an endvertex of a Hamilton path of $H$ to $x_{14}$, so $N\left(x_{5}\right) \subseteq V(C)$. By $\left(Q_{2}\right), x_{5}$ has no neighbor in the cycle $x_{7} x_{8} \cdots x_{13} x_{7}$. By $\left(Q_{1}\right), x_{5}$ has extra neighbors only in $\left\{x_{1}, x_{3}, x_{14}\right\}$. If $x_{5}$ is adjacent to $x_{1}$, then $x_{2}$ is an endvertex of a Hamilton path of $H$ to $x_{14}$, by applying $\left(Q_{2}\right)$ to $x_{1} x_{2} x_{3}$ and the cycle $x_{4} x_{5} \cdots x_{13} x_{4}$, we have a contradiction; otherwise, $x_{5}$ is adjacent to $x_{3}$, then $\left\{x_{1}, x_{5}, x_{9}, x_{13}\right\}$ dominates $V(C)-\left\{x_{14}\right\}$, also a contradiction.

3. $x_{1}$ is adjacent to $x_{10}$. By $\mathbf{1}$ and $\mathbf{2}, x_{1}$ has one more neighbor only in $\left\{x_{4}, x_{5}, x_{7}, x_{8}\right\}$. In this case, $x_{9}$ is an endvertex of a Hamilton path of $H$ to $x_{14}$. Clearly, by applying $\left(Q_{2}\right)$, we have the following claim.

$$
x_{12} \text { has no neighbor in the cycle } x_{1} x_{2} \cdots x_{10} x_{1} \text {. }
$$

Then, $x_{9}, x_{13}$ are not adjacent, for otherwise $x_{12}$ is an endvertex of a Hamilton path of $H$ to $x_{14}$, and thus $N\left(x_{12}\right) \subseteq V(C)$, as $\delta(G) \geq 4$, contradicting $(F)$. Hence, by noting that $x_{13}, x_{1}$ are symmetrical, $x_{13}$ has extra neighbors only in $\left\{x_{10}, x_{7}, x_{6}, x_{4}\right\}$. Clearly, by $\left(Q_{1}\right), x_{9}$ is not adjacent to $x_{12}$. And also $x_{9}$ is not adjacent to $x_{11}$, for otherwise $x_{12}$ is an endvertex of a Hamilton path of $H$ to $x_{14}$, contradicting $(F)$. So, by $\left(Q_{1}\right), x_{9}$ has extra neighbor only in $\left\{x_{2}, x_{3}, x_{5}, x_{6}, x_{14}\right\}$. In the following we distinguish four subcases.

(3.1) $x_{1}$ is adjacent to $x_{4}$. Then, $x_{3}$ is an endvertex of a Hamilton path of $H$ to $x_{14}$. As $x_{1}$ dominates $\left\{x_{2}, x_{4}, x_{10}\right\}$, by $\left(Q_{3}\right)$ and $\left(Q_{1}\right)$ and above result, $x_{3}$ has extra neighbors only in $\left\{x_{7}, x_{10}, x_{11}, x_{14}\right\}$. If $x_{3}$ is adjacent to $x_{7}$, we apply $\left(Q_{2}\right)$ to $x_{8} x_{9} x_{10}$ and the cycle $x_{1} x_{2} x_{3} x_{7} \cdots x_{4} x_{1}$ to obtain a contradiction. If $x_{3}$ is adjacent to both $x_{11}, x_{14}$, then it is the same situation as $\mathbf{2}$, a contradiction. If $x_{3}$ is adjacent to both $x_{10}, x_{11}$, then $x_{2}$ is an endvertex of a Hamilton path of $H$ to $x_{14}$. Thus, we can similarly deduce that $x_{2}$ has extra neighbors only in $\left\{x_{5}, x_{8}, x_{11}, x_{14}\right\}$, and hence $\left\{x_{5}, x_{8}, x_{11}, x_{14}\right\}$ dominates $V(C)-\left\{x_{14}\right\}$, a contradiction. So, $x_{3}$ must be adjacent to both $x_{10}, x_{14}$. Next we check the neighbors of $x_{9}$.

By $\left(Q_{2}\right), x_{9}$ has no neighbors in the cycle $x_{1} x_{2} x_{3} x_{4} x_{1}$. Then, $x_{9}$ has extra neighbors only in $\left\{x_{5}, x_{6}, x_{14}\right\}$. First assume that $x_{9}$ is adjacent to $x_{5}$. Then, $x_{13}$ is not adjacent to $x_{7}$, for otherwise $\left\{x_{2}, x_{5}, x_{7}, x_{11}\right\}$ dominates $V(C)-\left\{x_{14}\right\}$, a contradiction. Moreover, if $x_{13}$ is adjacent to $x_{6}$, then 
$x_{12} x_{11} x_{10} x_{1} \cdots x_{5} x_{9} \cdots x_{6} x_{13} x_{14}$ is a Hamilton path of $H$, contradict $(F)$. Hence, in this case, $x_{13}$ is adjacent to both $x_{4}, x_{10}$. Note that

$$
x_{6} \cdots x_{9} x_{5} \cdots x_{1} x_{10} \cdots x_{14}
$$

is a Hamilton path of $H$, we have $N\left(x_{6}\right) \subseteq V(C)$. We can similarly deduce that $x_{6}$ has extra neighbors only in $\left\{x_{9}, x_{10}, x_{14}\right\}$. If $x_{6}$ is adjacent to $x_{9}$, then $x_{7} x_{8} x_{9} x_{6} \cdots x_{1} x_{10} \cdots x_{14}$ is a Hamilton path of $H$; if $x_{6}$ is adjacent to $x_{10}$, then $x_{7} x_{8} x_{9} x_{5} x_{6} x_{10} \cdots x_{13} x_{4} x_{3} \cdots x_{14}$ is a Hamilton path of $H$. Thus, $N\left(x_{7}\right) \subseteq V(C)$. Now we apply $\left(Q_{2}\right)$ to the cycle $x_{1} \cdots x_{5} x_{9} x_{10} x_{1}$ and the cycle $x_{10} \cdots x_{13} \quad x_{10}$ to deduce that $x_{7}$ has only one extra neighbor $x_{14}$, a contradiction.

Hence, $x_{9}, x_{5}$ are not adjacent, and thus $x_{9}$ is adjacent to both $x_{6}, x_{14}$. Now if $x_{13}$ is adjacent to $x_{7}$, then $x_{12} x_{11} x_{10} x_{1} \cdots x_{6} x_{9} x_{8} x_{7} x_{13} x_{14}$ is a Hamilton path of $H$, contradict $(F)$. So, $x_{13}$ has extra neighbors only in $\left\{x_{4}, x_{6}, x_{10}\right\}$. If $x_{13}$ is adjacent to both $\left\{x_{4}, x_{10}\right\}$, by symmetry, we can similarly deduce that $x_{5}$ is adjacent to both $x_{8}, x_{14}$, and thus $\left\{x_{3}, x_{7}, x_{11}, x_{14}\right\}$ dominates $V(C)-$ $\left\{x_{14}\right\}$, a contradiction. If $x_{13}$ is adjacent to both $\left\{x_{4}, x_{6}\right\}$, by noting that $x_{13}$ dominates $\left\{x_{4}, x_{6}, x_{12}\right\}$ and that both $x_{9}, x_{12}$ are not adjacent to $x_{5}$, we can deduce that $x_{5}$ has only one extra neighbor $x_{14}$, a contradiction. If $x_{13}$ is adjacent to both $\left\{x_{6}, x_{10}\right\}$, then $x_{5} \cdots x_{1} x_{10} \cdots x_{13} x_{6} \cdots x_{9} x_{14}$ is a Hamilton path of $H$. By $\left(Q_{1}\right), x_{5}$ is not adjacent to $x_{8}$. If $x_{5}$ is adjacent to $x_{7}$ or $x_{14}$, then $\left\{x_{3}, x_{7}, x_{11}, x_{14}\right\}$ dominates $V(C)-\left\{x_{14}\right\}$, a contradiction. As $x_{5}$ is not adjacent to $x_{9}$, then $x_{5}$ has a neighbor in the cycle $C_{1}^{\prime}:=x_{1} x_{2} x_{3} x_{10} x_{1}$ or the cycle $C_{2}^{\prime}:=x_{10} x_{11} x_{12} x_{13} x_{10}$. By applying $\left(Q_{2}\right)$ to $x_{4} x_{5} x_{6}$ and $C_{1}^{\prime}$, or to $x_{4} x_{5} x_{6}$ and $C_{2}^{\prime}$, we have a contradiction.

By (3.1) and the symmetry of $x_{1}$ and $x_{13}, x_{13}$ is also not adjacent to both $x_{4}$ and $x_{10}$.

(3.2) $x_{1}$ is adjacent to $x_{5}$. In this case, if either $x_{13}$ is adjacent to $x_{4}$, or $x_{13}$ is adjacent to both $x_{6}, x_{7}$, then $x_{12}$ is an endvertex of a Hamilton path of $H$ to $x_{14}$, contradict $(F)$. So, we only need check for that $x_{13}$ is adjacent to both $x_{6}, x_{10}$, or to both $x_{7}, x_{10}$.

First, let $x_{13}$ be adjacent to both $x_{6}, x_{10}$. Then, $x_{11}$ is an endvertex of a Hamilton path of $H$ to $x_{14}$. As $x_{13}$ dominates $\left\{x_{6}, x_{10}, x_{12}\right\}$, if $x_{11}$ has one neighbor in the cycle $x_{1} \cdots x_{5} x_{1}$, then we can easily find four vertices to dominate $V(C)-\left\{x_{14}\right\}$. On the other hand, if $x_{11}$ is adjacent to $x_{7}$, then $x_{12} x_{11} x_{7} \cdots x_{10} x_{13} x_{6} \cdots x_{1} x_{14}$ is a Hamilton path of $H$, contradict $(F)$. Clearly, $x_{11}$ is not adjacent to $x_{8}, x_{9}, x_{13}$, and thus $x_{11}$ is adjacent to both $x_{6}, x_{14}$. Then, $x_{2} \cdots x_{5} x_{1} x_{10} \cdots x_{6} x_{11} \cdots x_{14}$ is a Hamilton path of $H$. By $\left(Q_{2}\right), x_{2}$ has no neighbor in the cycle $C_{3}^{\prime}:=x_{10} \cdots x_{13} x_{10}$. If $x_{2}$ is adjacent to any vertex in $\left\{x_{5}, x_{6}, x_{9}\right\}$, then $x_{3}$ is an endvertex of a Hamilton path of $H$ to $x_{14}$. Thus, by applying $\left(Q_{2}\right)$ to $x_{2} x_{3} x_{4}$ the cycle $x_{1} x_{5} \cdots x_{10} x_{1}$ and $C_{3}^{\prime}$, we have a contradiction. Otherwise, by $\left(Q_{1}\right), x_{2}$ is adjacent to $x_{8}$. Then $\left\{x_{4}, x_{8}, x_{10}, x_{13}\right\}$ dominates $V(C)-\left\{x_{14}\right\}$, a contradiction. 
Secondly, let $x_{13}$ be adjacent to both $x_{7}, x_{10}$. Then, $x_{8}$ is an endvertex of a Hamilton path of $H$ to $x_{14}$. By applying $\left(Q_{2}\right)$ to $x_{7} x_{8} x_{9}$ and the cycle $C_{3}^{\prime}$, we have that $x_{8}$ has no neighbor in $C_{3}^{\prime}$. As $x_{10}$ dominates $x_{1}, x_{9}, x_{11}$, we apply $\left(Q_{3}\right)$ to $x_{8}$ and the path $x_{12} x_{13} x_{7} \cdots x_{2}$, then $x_{8}$ has extra neighbors only in $\left\{x_{14}, x_{4}, x_{1}\right\}$. Then, $x_{8}$ is adjacent to $x_{1}$ or $x_{4}$, and thus, $x_{12}$ is an endvertex of a Hamilton path of $H$ to $x_{14}$, contradict $(F)$.

(3.3) By above, $x_{1}$ is adjacent to $x_{7}$ or $x_{8}$. If $x_{1}$ is adjacent to $x_{7}$, then we apply $\left(Q_{2}\right)$ to $x_{8} x_{9} x_{10}$ and the cycle $x_{1} \cdots x_{7} x_{1}$ to deduce a contradiction. If $x_{1}$ is adjacent to $x_{8}$, then, by $(F)$ and $\left(Q_{3}\right)$, we can deduce that $x_{13}$ is adjacent to both $x_{4}, x_{10}$, as above claim, also a contradiction.

4. $x_{1}$ is adjacent to $x_{8}$. From 1-3 and the symmetry, $x_{13}$ has extra neighbors only in $\left\{x_{6}, x_{7}, x_{9}, x_{10}\right\}$. If $x_{13}$ is adjacent to $x_{6}$, then $\left\{x_{3}, x_{6}, x_{8}, x_{11}\right\}$ dominates $V(C)-\left\{x_{14}\right\}$, a contradiction. Otherwise, as $x_{13}$ is not adjacent to both $x_{9}, x_{10}$, then $x_{13}$ is adjacent to $x_{7}$, then $x_{2}$ is an endvertex of a Hamilton path of $H$ to $x_{14}$. By applying $x_{1} x_{2} x_{3}$ and the cycle $x_{7} \cdots x_{13} x_{7}$, we know that $x_{7}$ has no neighbor in this cycle. If $x_{2}$ is adjacent to both $x_{5}, x_{6}$ Then, $x_{4}$ is an endvertex of a Hamilton path $H$ to $x_{14}$, by $\left(Q_{2}\right)$ (for $x_{3} x_{4} x_{5}$ and the cycle $\left.x_{1} x_{2} x_{6} x_{7} x_{13} \cdots x_{8} x_{1}\right)$, we have a contradiction. Otherwise, $x_{2}$ must be adjacent to $x_{14}$, this is the same situation as $\mathbf{1}$, a contradiction.

5. From 1-4 and the fact $x_{1}$ is not adjacent to both $x_{4}, x_{5}$, we have that $x_{1}$ is adjacent to $x_{7}$. Symmetrically, $x_{13}$ is also adjacent to $x_{7}$. On the other hand, $x_{1}$ has one more neighbor in $\left\{x_{4}, x_{5}\right\}$. First let $x_{1}$ be adjacent to $x_{4}$. As $x_{6}$ is an endvertex of a Hamilton path of $H$ to $x_{14}$, we can similarly use $\left(Q_{1}\right)$ and $\left(Q_{2}\right)$ to deduce that, $x_{6}$ has extra neighbors only in $\left\{x_{8}, x_{10}, x_{11}, x_{14}\right\}$. As the former cases shown, $x_{6}$ is not adjacent to $x_{14}$. Thus, $x_{6}$ is adjacent to either $x_{8}$, or both $x_{10}, x_{11}$. If $x_{6}$ is adjacent to $x_{8}$, then $x_{12}$ is an endvertex of a Hamilton path of $H$ to $x_{14}$, and thus, by $\left(Q_{2}\right)$ and $\left(Q_{1}\right)$ and 1 shown, $x_{12}$ is adjacent to both $x_{8}, x_{9}$. Then, $x_{10}$ is an endvertex of a Hamilton path of $H$ to $x_{14}$. Again by $Q_{2}$ for $x_{9} x_{10} x_{11}$ and the cycle $x_{1} \cdots x_{8} x_{12} x_{13} x_{1}$, we have a contradiction. Otherwise, $x_{6}$ is adjacent to both $x_{10}, x_{11}$. Then, $x_{9}$ is an endvertex of a Hamilton path of $H$ to $x_{14}$. By $\left(Q_{2}\right)$ for $x_{8} x_{9} x_{10}$ and the cycle $x_{1} \cdots x_{6} x_{11} x_{12} x_{13} x_{7} x_{1}$, we also have a contradiction.

Hence, $x_{1}$ is adjacent to $x_{5}$, and by symmetry, $x_{13}$ is adjacent to $x_{9}$. As $x_{6}$ is an endvertex of a Hamilton path of $H$ to $x_{14}$, by $\left(Q_{1}\right), x_{6}$ has extra neighbors only in $\left\{x_{2}, x_{3}, x_{8}, x_{10}, x_{11}, x_{14}\right\}$. If $x_{6}$ is adjacent to $x_{3}$ or $x_{11}$, then $\left\{x_{1}, x_{3}, x_{9}, x_{12}\right\}$ or $\left\{x_{1}, x_{4}, x_{9}, x_{11}\right\}$ dominates $V(C)-\left\{x_{14}\right\}$, a contradiction. If $x_{6}$ is adjacent to $x_{2}$, as $x_{4}$ is an endvertex of a Hamilton path of $H$ to $x_{14}$, by $\left(Q_{2}\right)$ for $x_{3} x_{4} x_{5}$ and the cycles $x_{1} x_{2} x_{6} x_{7} x_{1}$ and $x_{7} \cdots x_{13} x_{7}$, we have a contradiction. Hence, $x_{6}$ has extra neighbors only in $\left\{x_{8}, x_{10}, x_{14}\right\}$, and thus, by the former cases shown, $x_{6}$ is adjacent to both $x_{8}, x_{10}$. Then, $x_{12}$ is an endvertex of a Hamilton path of $H$ to $x_{14}$, then, by $\left(Q_{2}\right)$ for $x_{11} x_{12} x_{13}$ and the cycle $x_{1} \cdots x_{6} x_{10} \cdots x_{7} x_{1}$, we also have a contradiction. This proves Lemma 2. 
A lasso $L$ is defined as a graph by identifying one vertex in a cycle $C$ with an endvertex of a path $P$. The other endvertex of the path $P$ is called the end of $L$, the common vertex of $C$ and $P$ is called the connecting vertex (sometimes, with a little abuse, we also regard a cycle as a lasso). Now we use Lemma 1 and Lemma 2 to deduce the results we need.

Lemma 3. Let $P \in S$ be a 2-path with at most one out-endvertex. If $|P| \leq 8$, then $V(P)$ has a subset of $\left\lfloor\frac{|P|}{3}\right\rfloor$ vertices which dominate all vertices of $V(P)$ except for the possible out-endvertex.

Proof. Clearly, Lemma 3 implies Assertion 2. If $|P|=2$, then $P$ has two outendvertices. If $|P|=5$ and $P$ has at most one out-endvertex, it is also easy to verify that $P$ can be dominated by one vertex. Now let $P=x_{1} x_{2} \cdots x_{8}$. Let $H$ be the subgraph induced by $V(P)$. If $|G|=8$, then $G=H$. As each vertex of $G$ has degree at least four, we can verify directly that $H$ can be dominated by two vertices. So, let $|G|>8$. We first claim that $P$ has one out-endvertex. Otherwise, both $x_{1}$ and $x_{8}$ have at least four neighbors in $V(P)$, from that it is easy to see that $H$ has a Hamilton cycle. As $G$ is connected, there exists at least one edge joining $V(P)$ and $V(G)-V(P)$. By our choices to maximize the number of the out-endvertices of $S$. This is a contradiction. Hence, $P$ has precisely one out-endvertex.

Let $x_{8}$ be the out-endvertex of $P$. We choose a lasso $L$ with $x_{8}$ as the end of $L$, such that the cycle of $L$ has maximum length. For convenience, we denote the vertices of $L$ along a Hamilton path of $L$ from the end as $x_{8}, x_{7}, \ldots, x_{1}$. Let $v$ be the connecting vertex. Clearly, $x_{1}$ is adjacent to $v$. Let $C^{\prime}=x_{1} x_{2} \cdots v x_{1}$. By $\left(Q_{1}\right), v=x_{3 k+1}$ or $v=x_{3 k+2}(1 \leq k \leq 2)$. By the choice of $L, C^{\prime}$ satisfies the condition of Lemma 1 or Lemma 2. By Lemma 1 or Lemma 2, we can deduce that $V(P)-\left\{x_{8}\right\}$ can be dominated by two vertices.

Lemma 4. Let $P \in S$ be a 1-path with no out-endvertex. If $|P| \leq 19$, then $P$ can be dominated by $\left\lfloor\frac{|P|}{3}\right\rfloor$ vertices.

Proof. We first prove that, if $|G|=|P|=19$ and $G$ has a Hamilton cycle, then $G$ has a dominating set of 8 vertices. Let $C=b_{1} b_{2} \cdots b_{19} b_{1}$ be a Hamilton cycle of $G$. Assume the conclusion is not true. By $\left(Q_{1}\right), b_{1}$ is not adjacent to $b_{3 k}(1 \leq k \leq 8)$. Note that $b_{1}$ is adjacent to $b_{5}$, for otherwise, by applying $\left(Q_{2}\right)$ for $b_{2} b_{3} b_{4}$ and the cycle $b_{1} b_{5} \cdots b_{19} b_{1}$, we have a contradiction. Similarly, $b_{3}$ is also not adjacent to any of $b_{5}, b_{7}$. If $b_{1}$ is adjacent to $b_{8}$, then, by $\left(Q_{2}\right)$ for $b_{2} b_{3} b_{4}$ and the cycle $b_{1} b_{8} \cdots b_{19} b_{1}, b_{3}$ has no neighbor in this cycle, and thus, $b_{3}$ must be $b_{5}$ or $b_{7}$, a contradiction. So, $b_{1}$ is also not adjacent to $b_{8}$. By symmetry, $b_{1}$ is not adjacent to any of $x_{16}, x_{13}$. Clearly, for each $2 \leq i \leq 19$, $b_{i}$ has the similar property as $b_{1}$. Now, if $b_{1}$ is adjacent to $b_{4}$, as $b_{1}$ dominates $b_{2}, b_{4}, b_{19}$, by $\left(Q_{3}\right), b_{3}$ has extra neighbors only in $\left\{b_{13}, b_{16}, b_{19}\right\}$; as $b_{4}$ dominates $b_{1}, b_{3}, b_{5}$, by $\left(Q_{3}\right), b_{2}$ has extra neighbors only in $\left\{b_{5}, b_{8}, b_{11}\right\}$. Since $\delta(G) \geq 4$, $\left\{b_{5}, b_{8}, b_{11}, b_{13}, b_{16}, b_{19}\right\}$ dominates $V(G)$, a contradiction. Hence, $b_{1}$ is also not adjacent to $b_{4}$, symmetrically, not adjacent to $b_{17}$. And hence, $b_{1}$ is not 
adjacent to $b_{11}$, for otherwise, we look at $b_{6}$, as $b_{6}$ has similar properties as $b_{1}$, then $b_{6}$ must have neighbors in the cycle $b_{1} b_{11} \cdots b_{19} b_{1}$, and thus, by applying $\left(Q_{2}\right)$ for $b_{5} b_{6} b_{7}$ and this cycle, we have a contradiction. Symmetrically, $b_{1}$ is not adjacent to $b_{10}$. So, $b_{1}$ is adjacent to both $x_{7}, x_{14}$. Similarly, $b_{3}$ is adjacent to both $b_{9}, b_{16}$. Then, $\left\{b_{1}, b_{5}, b_{9}, b_{12}, b_{15}, b_{18}\right\}$ dominates $V(G)$, a contradiction. So, in this case, $G$ has a dominating set of 8 vertices.

Next we assume that either $|G|>19$ or $G$ has no hamiltonian cycle. Let $|P|=3 m+1(1 \leq m \leq 6)$. Let $H$ be the subgraph induced by $V(P)$. Now we claim that $H$ has no Hamilton cycle. For otherwise, if $|G|>|P|$, as $G$ is connected, then $P$ has at least one out-endvertex, a contradiction; if $|G|=$ $|P|=19$, then $G=H$, contradicting that $G$ has no Hamilton cycle. Now we choose a lasso $L$ in $H$ such that the number of vertices on the cycle of the lasso is maximum. For convenience, we label the vertices of $L$ along a Hamilton path on $L$ from the end of $L$ as $x_{3 m+1}, x_{3 m}, \ldots, x_{1}$. Let $v$ be the connecting vertex. By the labelling, $x_{1}$ is adjacent to $v$. By $\left(Q_{1}\right)$ and our assumption, we may assume that $v=x_{3 k+1}$ or $x_{3 k+2}(1 \leq k<m)$. If $k \leq 4$, then, by the choice of $L$, the cycle $C^{\prime}=x_{1} x_{2} \cdots v x_{1}$ satisfies the condition of Lemma 1 or Lemma 2, and thus we can obtain the desired result by Lemma 2 or Lemma 3. So, we next let $k \geq 5$. Hence, $m=6, k=5$ and $|P|=19$. We still prove by contradiction.

Case 1. $v=x_{3 k+1}=x_{16}$. Denote the cycle $C^{\prime}:=x_{1} x_{2} \cdots x_{16} x_{1}$. By the choice of $L, N\left(x_{1}\right) \subseteq V\left(C^{\prime}\right)$ and $N\left(x_{15}\right) \subseteq V\left(C^{\prime}\right)$. We can similarly as in the proof of Lemma 1 deduce the following.

(F) $\quad x_{1}$ is not adjacent to both $x_{4}, x_{5} ; x_{15}$ is not adjacent to both $x_{9}, x_{10}$.

By $\left(Q_{2}\right), x_{1}$ is not adjacent to $x_{13}$. If $x_{1}$ is adjacent to $x_{10}$, then, by $\left(Q_{2}\right), x_{15}$ has no neighbor in the cycle $x_{1} \cdots x_{10} x_{1}$, and thus, by $\left(Q_{1}\right), x_{15}$ is adjacent to both $x_{9}, x_{10}$, a contradiction. So, $x_{1}$ is also not adjacent to $x_{10}$. Symmetrically, $x_{15}$ is not adjacent to any of $x_{4}, x_{7}$. Now, we check the possible neighbors of $x_{19}$.

Clearly, $N\left(x_{19}\right) \subseteq V(P)$. Moreover, by the choice of $L$ and $\left(Q_{1}\right), x_{19}$ has extra neighbors only in $\left\{x_{4}, x_{7}, x_{9}, x_{12}, x_{16}\right\} \cup\left\{x_{6}, x_{10}\right\}$. If $x_{19}$ is adjacent to $x_{6}$, we look at the Hamilton path $x_{1} \cdots x_{6} x_{19} \cdots x_{7}$ of $H$, by the choice of $L$ and $\left(Q_{1}\right)$, we can deduce that $x_{1}$ is adjacent to both $x_{4}, x_{5}$, contradict $(F)$. Note that $x_{6}$ and $x_{10}$ are symmetrical, we have $x_{19}$ is not adjacent to any of $x_{6}, x_{10}$. If $x_{19}$ is adjacent to both $x_{4}, x_{9}$, then we can similarly deduce that $x_{1}$ must be adjacent to both $x_{4}, x_{8}$, and thus $x_{2} x_{3} \cdots x_{1} x_{8} x_{16} x_{15} \cdots x_{9} x_{19} x_{18} x_{17}$ is a hamiltonian path of $H$. By applying $\left(Q_{2}\right)$ for $x_{1} x_{2} x_{3}$ and the cycle $x_{4} \cdots x_{19} x_{4}$, we have a contradiction. Hence, $x_{19}$ is adjacent to at most one of $x_{4}, x_{9}$, and symmetrically, is adjacent to at most one of $x_{7}, x_{12}$. So, $x_{19}, x_{16}$ are adjacent. Thus, $x_{17}$ is also an endvertex of a hamiltonian path of $H$. Then, $x_{17}, x_{19}$ have the same properties. 
Note that $x_{19}$ must be adjacent to one of $x_{4}, x_{9}$, and one of $x_{7}, x_{12}$. First let $x_{19}$ be adjacent to $x_{4}$. As $x_{17}$ is also adjacent to one of $x_{7}, x_{12}$, if $x_{17}$ is adjacent to $x_{4}$, then $\left\{x_{2}, x_{6}, x_{9}, x_{12}, x_{15}, x_{19}\right\}$ dominates $P$; if $x_{17}$ is adjacent to $x_{7}$, then $x_{7} \cdots x_{16} x_{1} \cdots x_{4} x_{19} x_{18} x_{17} x_{7}$ is a cycle of $H$ which exclude $x_{5}, x_{6}$, contradict the choice of $L$. Then, by symmetry, $x_{19}$ is adjacent to both $x_{7}, x_{9}$. Also, $x_{17}$ is adjacent to these two vertices. Hence, $x_{1} \cdots x_{7} x_{19} x_{18} x_{17} x_{9} \cdots x_{16} x_{1}$ is a cycle of $H$ which exclude $x_{8}$, contradict the choice of $L$.

Case 2. $v=x_{3 k+2}=x_{17}$. By $\left(Q_{1}\right)$ and the choice of $L, x_{19}$ is not adjacent to any of $x_{17}, x_{1}, x_{16}$. As $x_{17}$ dominates $x_{18}, x_{1}, x_{16}$, by $\left(Q_{3}\right), x_{19}$ has extra neighbors only in $\left\{x_{4}, x_{7}, x_{10}, x_{13}\right\}$. Since $x_{19}$ is adjacent to three of them, by symmetry, we assume that $x_{19}$ is adjacent to both $x_{4}, x_{7}$. Then, $x_{5}$ is an endvertex of a Hamilton path of $H$, and thus $N\left(x_{5}\right) \subseteq V(P)$. As $x_{7} \cdots x_{19} x_{7}$ is a 13-cycle, by looking at $x_{4} x_{5} x_{6}$ and this cycle, we deduce that $x_{5}$ has no neighbor in this cycle. So, $x_{5}$ has extra neighbors only in $\left\{x_{1}, x_{2}, x_{3}\right\}$. If $x_{5}$ is adjacent to $x_{3}$, then $\left\{x_{5}, x_{1}, x_{15}, x_{12}, x_{9}, x_{19}\right\}$ dominates $P$; otherwise $x_{5}$ is adjacent to both $x_{1}, x_{2}$, then $\left\{x_{4}, x_{5}, x_{8}, x_{11}, x_{14}, x_{17}\right\}$ dominates $P$, a contradiction. This proves Lemma 4.

Lemma 5. Let $T \in E^{\prime}$ be a tip of a 2-path $P$ in $A$. If $|T| \leq 13$, then $T$ can be dominated by $\left\lfloor\frac{|T|}{3}\right\rfloor$ vertices.

Proof. Let $T=a_{0} \cdots a_{3 m+1} \in E^{\prime}(m \leq 4)$ and $C=c_{0} \cdots c_{l}$ be the central path of $P$. Assume that $c_{0}$ is adjacent to $a_{3 m+1}$ on the path $P$. By definition, $c_{1}$ is an acceptor or inacceptor. As $T \in E^{\prime}$, neither endpoint of $P$ is $(2,2)$-endpoint, and thus $c_{1}$ is an acceptor. We first present a claim (for the proof, see [11, p. 285, Fact 11]).

Claim. $\quad a_{0}$ is only adjacent to the vertices of $T \cup\left\{c_{0}\right\}$.

By the choice of $S$ and the claim, if $a_{0}^{\prime} \cdots a_{3 m+1}^{\prime}$ is a Hamilton path on $V(T)$ such that $a_{3 m+1}^{\prime}$ is adjacent to $c_{0}$, then $a_{0}^{\prime}$ is also only adjacent to the vertices of $T \cup\left\{c_{0}\right\}$.

Let $H$ be the subgraph induced by $V(T) \cup\left\{c_{0}\right\}$. We choose a lasso $L$ with $c_{0}$ as the end of $L$, such that the cycle of $L$ has maximum length. For convenience, we denote the vertices of $L$ along a hamiltonian path of $L$ from $c_{0}$ as $x_{3 m+2}, x_{3 m+1}, \ldots, x_{1}$. Let $v$ be the connecting vertex. Clearly, $x_{1}$ is adjacent to $v$. Let $C^{\prime}=x_{1} x_{2} \cdots v x_{1}$. By $\left(Q_{1}\right), v=x_{3 k+1}$ or $v=x_{3 k+2}(1 \leq k \leq m \leq 4)$. By the choice of $L, C^{\prime}$ satisfies the conditions of Lemma 1 or Lemma 2. By Lemma 1 or Lemma 2, we can deduce the desired result.

Proof of Main Theorem. By Lemma 4, $|P| \geq 22$ for any path $P$ in $I_{1}$. By Lemma $3,|P| \geq 11$ for any path $P$ in $I_{2}$. Hence,

$$
\sum_{P \in I_{1}}|P| \geq 22\left|I_{1}\right| ; \quad \sum_{P \in I_{2}}|P| \geq 11\left|I_{2}\right|
$$


By Lemma 5,

From that we have,

$$
\sum_{P \in A}|P| \geq 17\left|E^{\prime}\right|
$$

$$
n \geq \sum_{P \in I_{1}}|P|+\sum_{P \in I_{2}}|P|+\sum_{P \in A}|P| \geq 22\left|I_{1}\right|+11\left|I_{2}\right|+17\left|E^{\prime}\right|,
$$

yielding, $\frac{n}{33} \geq \frac{2}{3}\left|I_{1}\right|+\frac{1}{3}\left|I_{2}\right|+\frac{1}{2}\left|E^{\prime}\right|$. Combining with $(*)$, we have $|D| \leq \frac{4}{11} n$. This proves Main Theorem.

Remark. We have verified by the same method, that the conjecture for the remaining two cases $k=5,6$ is also true.

\section{References}

[1] N. Alon and J. H. Spencer, The Probabilistic Method, Wiley-Interscience, New York, 1992.

[2] Y. Caro and Y. Roditty, On the vertex-independence number and star decomposition of graphs, Ars Combin. 20 (1985), 167-180.

[3] - A note on the $k$-domination number of a graph, Internat. J. Math. Math. Sci. 13 (1990), no. 1, 205-206.

[4] G. Chartrand and L. Lesniak, Graphs and Digraphs, 3rd Edition, Chapman and Hall, London, 1996.

[5] M. R. Garey and D. S. Johnson, Computers and intractability, A guide to the theory of NP-completeness. A Series of Books in the Mathematical Sciences. W. H. Freeman and Co., San Francisco, Calif., 1979.

[6] T. W. Haynes, S. T. Hedetniemi, and P. J. Slater, Fundamentals of Domination in Graphs, Marcel Dekker, Inc., New York, 1998.

[7] _ Domination in Graphs, Marcel Dekker, Inc., New York, 1998.

[8] W. McCuaig and B. Shepherd, Domination in graphs with minimum degree two, J. Graph Theory 13 (1989), no. 6, 749-762.

[9] O. Ore, Theory of Graphs, American Mathematical Society Colloquium Publications, Vol. XXXVIII, American Mathematical Society, Providence, R.I. 1962.

[10] C. Payan and N. H. Xuong, Domination-balanced graphs, J. Graph Theory 6 (1982), no. 1, 23-32.

[11] B. A. Reed, Paths, stars and the number three, Combin. Probab. Comput. 5 (1996), no. $3,277-295$.

[12] _ Paths, stars and the number three: the grunge, University of Waterloo, Technical Report, 1993.

[13] L. A. Sanchis, Bounds related to domination in graphs with minimum degree two, J. Graph Theory 25 (1997), no. 2, 139-152.

Moo Young SoHn

Department of Applied Mathematics

Changwon National University

Changwon, 641-773, Korea

E-mail address: mysohn@changwon.ac.kr 
YUAN XUdONG

Department of Mathematics

Guangxi Normal University

541004, Guilin, P. R. China

E-mail address: yuanxd@public.glptt.gx.cn 